\title{
Quantitative Probiotic Analysis of Various Kefir Samples
}

\author{
Aylin USKUDAR GUCLU ${ }^{1, *}$, Esen YESIL ${ }^{2}$, Aylin ALTAY KOCAK ${ }^{1}$, Mendane \\ SAKA $^{2}$, Hasan Cenk MIRZA ${ }^{1}$, Bedia DINC ${ }^{3}$, Ahmet BASUSTAOGLU ${ }^{1}$ \\ ${ }^{1}$ Department of Medical Microbiology, Medical Faculty, Baskent University, Ankara, Turkey; ${ }^{2}$ Department of Nutrition and Dietetics, Health \\ Science Faculty, Baskent University, Ankara, Turkey; ${ }^{3}$ Department of Medical Microbiology, Ankara City Hospital, Ministry of Health,
} Ankara, Turkey,

Received: September 28, 2020; Revised: January 15, 2021; Accepted: January 30, 2021

\begin{abstract}
This study assessed whether industrial kefirs have enough probiotic diversity and number, which is important in health benefits, to examine the difference between fruit kefir (FK) and plain kefir (PK) products in terms of probiotic content, and to reveal how much they preserve the probiotic content until the expiry date. The number of total aerobic mesophilic bacteria (TAMB), Lactobacillus, Lactococcus, Leuconostoc, acetic acid bacteria, and yeast was determined using Standard Plate Count Agar, MRS (De Man, Rogosa and Sharpe) agar, M17 agar, MSE (Mayeux, Sandine \& Elliker) agar, Acetobacter agar and Sabouraud dextrose agar with chloramphenicol. Matrix-assisted laser desorption/ionization-time of flight mass spectrometry (MALDI-TOF-MS) and API 20AUX were used to identify the isolates. TAMB in PK was 4.3x105 to 2.9x108; in FK, 1.8x106 to 3.6x108. Lactobacillus paracasei, L.casei, Lactococcus lactis, Leuconostoc mesenteroides, Leuconostoc spp, Streptococcus spp, and Candida kefyr were isolated in different combinations and number. Acetic acid bacteria were not isolated. There was no difference in FK and PK of the same trademarks. The number of lactic acid bacteria (LAB) in FK and PK at two different times was similar. Total LAB, lactococci, lactobacilli, and yeast populations increased during storage $(\mathrm{p}<0.05)$. Although the number of microorganisms of samples decreased at expiration dates, they still had enough microorganism numbers indicated in a codex.
\end{abstract}

Keywords: Industrial Kefir, Probiotics, Microbial Composition, Fruit Kefir

\section{Introduction}

Kefir is a fermented-milk beverage produced by the action of lactic acid bacteria, yeasts, and acetic acid bacteria on milk (Ahmed et al., 2013). It originated in the Caucasus Mountains in ancient times and spread from there throughout the world. Kefir is produced by mesophilic bacteria and yeasts as a result of fermenting the lactic acid and alcohol (John and Deeseenthum, 2015). Recently, kefir has strong effects on health as a probiotic food like other fermented milk and dairy products; due to the organic acids, $\mathrm{H} 2 \mathrm{O} 2$, acetaldehyde, $\mathrm{CO} 2$, and bacteriocins produced as natural metabolites of microorganisms in its components, kefir shows antibacterial activity against many pathogenic microorganisms (Yuksekdag et al., 2004). In addition to its antibacterial and antifungal activities, kefir reduces cholesterol levels in serum and has antitumor, antiinflammatory, and immunomodulatory effects due to its content of Lactobacillus and Lactococcus (Otles and Cagindi, 2003; Vinderola et al. 2005; Prado et al. 2015). It was also reported that acetic acid, polysaccharides, and other chemicals produced by kefir microbial components, effectively wound healing (Hassan et al. 2012). Several studies have shown the positive effect of using probiotic foods, such as kefir, for treating many gastrointestinal disorders, such as diarrhea, irritable bowel syndrome, colitis, Crohn's disease, traveler's diarrhea, and chronic constipation (Reid et al. 2003; Heyman 2000; Maeda et al., 2004). In pregnant women, kefir consumption prevents the reproduction of Streptococcus agalactia strains that cause sepsis, pneumonia, and meningitis in newborns (John and Deeseenthum, 2015).

There are several methods for producing kefir, in which traditional and industrial processes are widely used. Industrial kefir can be prepared in high volumes by using a starter culture. No matter what method is used, the most important factor affecting kefir characteristics is the number of microorganisms and the ratio of species in the content, where the microbial composition varies depending on the source of kefir grains, the country of origin, and the starter culture used (Guzel-Seydim, 2005). The probiotic content that should be found in most kefir products is expressed in numerical values with no description of its microbial diversity. This study assesses whether kefir products have desired probiotic microorganism diversity and number, examine whether there is a difference between fruit kefir and plain kefir products in terms of probiotic content, and reveal how much they preserve the probiotic content until the expiration date. Thus, the necessity of including individuals in nutrition programs can be demonstrated with scientific data through the outputs and results to be obtained in this study.

\footnotetext{
* Corresponding author e-mail: uskudaraylin@gmail.com.
} 


\section{Materials and Methods}

\subsection{Data Collection}

This study was supported by Baskent University Research Fund (Project No.DA19/12). This study has examined 9 industrial kefir samples and 1 kefir sample fermented using kefir grains. In this study, we examined 4 different fruit kefirs (FK) and 5 different plain kefirs (PK). For each type, 2 bottles of kefir were bought simultaneously. To prepare the traditional kefir, Pasteurized cow's milk was used to prepare kefir samples with kefir grains from Ankara University Faculty of Agriculture. A ratio of 1:30 weight/volume was used as grain to milk ratio. After adding kefir grains to milk, kefir samples were produced in a closed container at a temperature of $20-25^{\circ} \mathrm{C}$ and the fermentation time was 24 h. The second measurement of kefir fermented using the traditional method was performed on the 7th day of fermentation.

\subsection{Microbiological Analysis}

Kefir samples were transferred to sterile tubes, and $\mathrm{pH}$ values were measured by $\mathrm{pH}$ meter (Inolab, Germany) immediately after they were opened, and then serial dilutions were prepared. Standard plate count agar (SPCA) (Oxoid, UK), MRS (Merck, Germany), M17 (Merck, Germany), MSE (Biokar, France), Acetobacter agar and Sabouraud dextrose (BD Difco,) agar with chloramphenicol (Biokar, France) (SDCA) media were used to determine the number of total aerobic mesophilic bacteria, Lactobacillus, Lactococcus, Leuconostoc, acetic acid bacteria, and yeast, respectively. Acetobacter agar was prepared with 3g/L glycose (Merck, Germany), 10g/L yeast extract (Sigma, Germany), 10g/L calcium carbonate (Aromel, Turkey), and 15g/L agar (BD Bacto, USA). All culture procedures were performed in duplicate.

SPCA was incubated for $48 \mathrm{~h}$ at $30^{\circ} \mathrm{C}$; M17 agar for 18-24 hours at $30^{\circ} \mathrm{C}$ in $5 \% \mathrm{CO} 2$ atmosphere; MSE agar for 4 days at $22^{\circ} \mathrm{C}$; MRS agar for 5 days at $30^{\circ} \mathrm{C}$ in $5 \%$ CO2atmosphere; and SDCA for 5 days at $22^{\circ} \mathrm{C}$. Plates with 30-300 colonies were counted at the end of incubation, and the results were recorded in $\mathrm{cfu} / \mathrm{ml}$. The same procedures were applied to the expiration date of each kefir sample.

After counting the plates, five randomized colonies from each counted plate were taken to identify isolates. The Matrix-Assisted Laser Desorption/Ionization Time-ofFlight Mass Spectrometry (MALDI-TOF) (VITEK® MS, Biomerieux, France) was used to identify bacteria using direct transfer method according to the direction of the manufacturer. API 20AUX (Biomerieux, France) and conventional methods were used to identify yeast isolates.

\subsection{Statistical Analysis}

The Shapiro Wilk and Kolmogrov Smirnov tests were used to check whether the data obtained had a normal distribution. The Mann-Whitney $U$ test was used to compare variables without normal distribution. The paired samples t-test was used to compare the numbers of microorganisms measured at two different times. The Wilcoxon test was used to compare non-normally distributed numerical measurements at 2 different times. The data were evaluated using a statistical package program (SPSS 22.0, IBM SPSS, USA), and $\mathrm{p}<0.05$ was considered statistically significant.

\section{Results}

Table 1 shows the total number of aerobic mesophilic organisms in kefir samples, including five plain and four fruit kefirs and one fermented using kefir grains. The total number of aerobic mesophilic organisms in fruit kefir samples was between $1.8 \times 10^{6}$ and $3.6 \times 10^{8}$ on the packages' opening date (OD); the number of living organisms of the same samples varied between $5.2 \times 10^{4}$ and $1.5 \times 10^{7}$ on the expiration dates (ED). The total number of aerobic mesophilic organisms in plain kefir samples was between $4.3 \times 10^{5}$ and $2.9 \times 10^{8}$ on the OD; and varied between $4.5 \times 10^{4}$ and $1.9 \times 10^{7}$ on the ED (Table 1 ).

Table 1. Number of aerobic mesophilic bacteria $(\mathrm{cfu} / \mathrm{mL})$ and $\mathrm{pH}$ value of kefir samples.

\begin{tabular}{|c|c|c|c|c|c|c|c|}
\hline & & \multicolumn{3}{|c|}{$\begin{array}{l}\text { Total number of aerobic } \\
\text { mesophilic bacteria }\end{array}$} & \multicolumn{3}{|c|}{$\mathrm{pH}$ values } \\
\hline & & OD & ED & $p$ & OD & ED & $p$ \\
\hline \multirow{4}{*}{ FK } & $1 \mathrm{a}$ & $3.6 \times 10^{8}$ & $1.5 \times 10^{7}$ & $0.000^{\ddagger *}$ & 4.5 & 4.3 & $0.068^{Y}$ \\
\hline & $2 \mathrm{a}$ & $3.6 \times 10^{8}$ & $1.5 \times 10^{7}$ & $0.066^{\beta}$ & 4.6 & 4.4 & $0.059^{\beta}$ \\
\hline & За & $1.0 \times 10^{7}$ & $5.2 \times 10^{4}$ & $0.043^{\beta^{*}}$ & 4.5 & 4.3 & $0.039^{\beta^{*}}$ \\
\hline & $4 a$ & $1.8 \times 10^{6}$ & $6.2 \times 10^{4}$ & $0.62 €$ & 4.4 & 4.3 & $0.10 €$ \\
\hline \multirow{5}{*}{ PK } & $1 \mathrm{~b}$ & $2.6 \times 10^{8}$ & $1.3 \times 10^{6}$ & $0.999^{\circ}$ & 4.6 & 4.4 & $0.090^{\circ}$ \\
\hline & $2 b$ & $2.9 \times 10^{8}$ & $9.9 \times 10^{5}$ & & 4.6 & 4.4 & \\
\hline & $3 b$ & $6.0 \times 10^{7}$ & $4.5 \times 10^{4}$ & & 4.6 & 4.5 & \\
\hline & $4 \mathrm{~b}$ & $3.2 \times 10^{8}$ & $1.9 \times 10^{7}$ & & 4.6 & 4.5 & \\
\hline & $5 b$ & $4.3 \times 10^{5}$ & $2.6 \times 10^{5}$ & & 4.5 & 4.3 & \\
\hline TK & 1c & $2.0 \times 10^{9}$ & $7.8 \times 10^{8}$ & & 4.6 & 4.7 & \\
\hline
\end{tabular}

FK: fruit kefir, PK: plain kefir, TK: traditional kefir, OD: first opening date of the packages, ED: expiration dates, p¥: Difference between OD and ED, $p \beta$ : Difference between OD and ED of the same samples, p€: Difference between OD of different samples, p@: Difference between ED of different samples, ${ }^{*} \mathrm{p}<0.05$.

The total number of aerobic mesophilic organisms in the kefir sample fermented with kefir grains was $2 \times 10^{9}$ at the 24th hour of the fermentation but did not change after $24 \mathrm{~h}$ and decreased to $7.8 \times 10^{8}$ at the end of the seventh day of fermentation. The mean decrease in the total number of aerobic mesophilic organisms was $\log 1.63 \pm 0.43$ in fruit kefir samples and $\log 1.86 \pm 1.14$ in plain kefir samples.

A statistically significant difference was observed between the packages in terms of the average number of living organisms measured at OD and ED $(p<0.001)$, where the average number was significantly lower at ED than at OD. The average number of living organisms was $7.84 \log 10 \mathrm{cfu} / \mathrm{mL}$ (6918309) for OD and $6.21 \log 10$ $\mathrm{cfu} / \mathrm{mL}$ (1621810) for ED. The total number of aerobic mesophilic organisms on OD and ED was compared for fruit and plain kefir groups. Accordingly, there was no statistically significant difference between the counts of living organisms measured at two different times for the FK group produced using the industrial method ( $p=0.066$ ). However, a statistically significant difference was found for the PK group produced using the industrial method $(p=0.043)$. The average number of living organisms in the PK group was lower at ED than at OD. The FK and PK 
groups were compared for both ED and OD, and no statistically significant difference was observed between them ( $p=0.623 ; p=0.999)$ (Table 1$)$.

The $\mathrm{pH}$ values of the samples are given in Table 1 . There was no statistically significant difference between the $\mathrm{pH}$ values measured at two different measuring times $(p=0.068)$. Besides, the intragroup comparisons of $\mathrm{pH}$ values at $\mathrm{OD}$ and $\mathrm{ED}$ were examined for each kefir type (plain and fruit). Accordingly, there was no statistically significant difference between OD and ED's pH values for the FK group $(\mathrm{p}=0.059)$, but a statistically significant difference was found between the values for the PK group $(p=0.039)$.

Table 2 shows the number of Lactobacillus, Lactococcus, Leuconostoc, Streptococcus, and yeast isolates in kefir samples, and Table 3 shows the microbial diversity of kefir samples included in the study. Accordingly, there was a statistically significant difference between the two counts (OD and ED) in terms of Lactobacillus $\quad(p=0.018), \quad$ Lactococcus $\quad(p=0.005)$, Leuconostoc $(p=0.012)$, and Streptococcus $(p=0.021)$, where the average value was lower at ED than OD for all four bacterial groups. An analysis was conducted to determine the difference between the average values at OD and ED, and intragroup comparisons were evaluated for the FK and PK groups. Accordingly, only in the PK group, there was a statistically significant difference between the numbers of Lactococcus at OD and ED ( $\mathrm{p}=0.043)$. The logarithmic reduction of Lactococcus in plain kefir samples was found as $1.44 \pm 0.53$. Four different FK and PK groups' parameters were compared for both counting times, and no statistically significant difference was found between them ( $p>0.05)$. Table 3 represents the microbial ingredient of each kefir sample indicates the species distribution. Acetic acid bacteria were found in none of the kefir samples included in the study. Only two industrial kefir samples (1 plane and 1 fruit kefir of same brand mark) had yeast, and Table 2 presents the average yeast numbers. Accordingly, there was no statistically significant difference between the yeast counts at OD and ED ( $>0.05)$. The yeast isolates were identified as Candida kefyr.

Table 2. The number of each bacterial genus of kefir samples (cfu/mL)

\begin{tabular}{|c|c|c|c|c|c|c|c|c|c|c|c|c|c|c|c|c|}
\hline & & \multicolumn{3}{|c|}{ Lactobacillus } & \multicolumn{3}{|c|}{ Lactococcus } & \multicolumn{3}{|c|}{ Leuconostoc } & \multicolumn{3}{|c|}{ Streptococcus } & \multicolumn{3}{|l|}{ Candida } \\
\hline & & OD & ED & $p$ & OD & ED & $p$ & OD & ED & $p$ & OD & ED & $p$ & OD & ED & $p$ \\
\hline \multirow[t]{4}{*}{ FK } & $1 \mathrm{a}$ & 0 & 0 & \multirow{6}{*}{$\begin{array}{c}0.018^{\mathrm{Y}_{*}} \\
0.109^{\beta} \\
0.068^{\beta} \\
0.539^{€}\end{array}$} & $2.0 \times 10^{6}$ & $4.0 \times 10^{5}$ & & $1.2 \times 10^{6}$ & $5.5 \times 10^{6}$ & & $2.8 \times 10^{8}$ & $6.4 \times 10^{6}$ & \multirow{6}{*}{$\begin{array}{l}0.021^{\Psi_{*}} \\
0.141^{\beta} \\
0.080^{\beta} \\
0.999^{€}\end{array}$} & & & \multirow{6}{*}{$\begin{array}{l}0.465^{\boldsymbol{Y}_{*}} \\
0.655^{\beta} \\
0.180^{\beta}\end{array}$} \\
\hline & $2 \mathrm{a}$ & $2.4 \times 10^{4}$ & $1.2 \times 10^{6}$ & & $1.2 \times 10^{6}$ & $2.8 \times 10^{5}$ & & $1.2 \times 10^{6}$ & $5.5 \times 10^{6}$ & & $2.8 \times 10^{8}$ & $6.4 \times 10^{6}$ & & & & \\
\hline & За & $1.7 \times 10^{5}$ & $1 \times 10^{4}$ & & $6.4 \times 10^{5}$ & $1.0 \times 10^{4}$ & & 0 & 0 & & $6.4 \times 10^{5}$ & $1.0 \times 10^{4}$ & & $1.4 \times 10^{5}$ & $5.2 \times 10^{3}$ & \\
\hline & $4 a$ & $2.4 \times 10^{4}$ & $4 \times 10^{3}$ & & $2.4 \times 10^{4}$ & $9.0 \times 10^{3}$ & $0.005^{Y_{*}}$ & $2.4 \times 10^{4}$ & $1.0 \times 10^{3}$ & \multirow{4}{*}{$\begin{array}{c}0.012^{\Psi_{*}} \\
0.102^{\beta} \\
-0.068^{\beta} \\
0.902^{€} \\
0.902^{\complement}\end{array}$} & $2.1 \times 10^{8}$ & $2.4 \times 10^{8}$ & & & & \\
\hline \multirow[t]{5}{*}{ PK } & $1 b$ & 0 & 0 & & $5.4 \times 10^{7}$ & $2.3 \times 10^{6}$ & \multirow{2}{*}{$\begin{array}{r}-0.043^{\beta} \\
-0.086^{€}\end{array}$} & $1.3 \times 10^{8}$ & $7.8 \times 10^{5}$ & & $5.4 \times 10^{7}$ & $2.3 \times 10^{6}$ & & & & \\
\hline & $2 b$ & $1.0 \times 10^{8}$ & $5.9 \times 10^{4}$ & & $4.0 \times 10^{8}$ & $5.5 \times 10^{6}$ & & $5.8 \times 10^{5}$ & $2.6 \times 10^{4}$ & & $4.0 \times 10^{8}$ & $3.5 \times 10^{6}$ & & & & \\
\hline & $3 b$ & $1.7 \times 10^{7}$ & $1.4 \times 10^{6}$ & $0.268^{\odot}$ & $2.7 \times 10^{6}$ & $4.1 \times 10^{4}$ & $0.176^{\complement}$ & 0 & 0 & & $1.2 \times 10^{5}$ & $5.0 \times 10^{4}$ & $0.806^{\odot}$ & $1.2 \times 10^{5}$ & $5.9 \times 10^{5}$ & \\
\hline & $4 \mathrm{~b}$ & $9.0 \times 10^{7}$ & $1.8 \times 10^{7}$ & & $1.5 \times 10^{7}$ & $4.2 \times 10^{6}$ & & $4.1 \times 10^{7}$ & $6.0 \times 10^{6}$ & & $1.6 \times 10^{7}$ & $2.2 \times 10^{7}$ & & & & \\
\hline & $5 b$ & $2.6 \times 10^{5}$ & $9.1 \times 10^{4}$ & & $4.2 \times 10^{5}$ & $1.0 \times 10^{4}$ & & $3.0 \times 10^{5}$ & $5.0 \times 104$ & & $3.1 \times 10^{9}$ & $1.1 \times 10^{8}$ & & & & \\
\hline TK & $1 c$ & 0 & 0 & & $3.1 \times 10^{9}$ & $2.0 \times 10^{3}$ & & $2.0 \times 10^{4}$ & $2.0 \times 10^{3}$ & & 0 & 0 & & & & \\
\hline
\end{tabular}

FK: fruit kefir, PK: plain kefir, TK: traditional kefir, OD: first opening date of the packages, ED: expire dates, $\mathrm{p}^{\sharp}$ : Difference between OD and ET, $\mathrm{p}^{\beta}$ : Difference between OD and ET of the same samples, ${ }^{*} \mathrm{p}<0.05$

Table 3. Microbial diversity of each kefir samples

\begin{tabular}{|c|c|c|c|c|c|c|}
\hline Samples & & Lactobacillus & Lactococcus & Leuconostoc & Streptococcus & Candida \\
\hline \multirow{4}{*}{ FK } & $1 \mathrm{a}$ & & L. lactis & L.mesenteroides, Leuconostoc spp. & Streptococcus spp. & \\
\hline & $2 \mathrm{a}$ & L. paracasei, L. casei & L. lactis & L.mesenteroides, Leuconostoc spp. & Streptococcus spp. & \\
\hline & 3a & L. paracasei & L. lactis & & Streptococcus spp. & C. kefyr \\
\hline & 4a & L. paracasei & L. lactis & Leuconostoc spp. & Streptococcus spp. & \\
\hline \multirow{5}{*}{ PK } & $1 b$ & & L. lactis & L.mesenteroides, Leuconostoc spp. & Streptococcus spp. & \\
\hline & $2 b$ & L. paracasei, L. casei & L. lactis & L.mesenteroides, Leuconostoc spp. & Streptococcus spp. & \\
\hline & $3 b$ & L. paracasei & L. lactis & & Streptococcus spp. & C. kefyr \\
\hline & $4 \mathrm{~b}$ & L. paracasei & L. lactis & Leuconostoc spp. & Streptococcus spp. & \\
\hline & $5 b$ & L. casei & L. lactis & Leuconostoc spp. & Streptococcus spp. & \\
\hline TK & 1c & & L. lactis & Leuconostoc spp. & & \\
\hline
\end{tabular}

FK: fruit kefir, PK: plain kefir, TK: traditional kefir

\section{Discussion}

Kefir is an old and traditional drink with unique taste and properties. In vitro studies and animal experiments have shown that kefir has health benefits due to its probiotic microorganisms (Farnworth, 2005; Walsh et al.,
2016). As kefir is a fermented beverage produced by bacteria and yeasts, its composition is complex due to the high number and diversity of microorganisms and the variety of possible bioactive compounds produced during fermentation. The changes in the process of kefir production from fermentation to consumption are 
remarkable. For example, even the size of kefir grains used in production affects the product profile's $\mathrm{pH}$, viscosity, and microbiological properties (Farnworth, 2003). As an alternative to the traditional method using kefir grains and a fermentation period of 20-24 h, industrial kefirs with acceptable kefir flavor and longer shelf life, which are produced using starter with lactic acid bacteria and yeast species isolated from kefir grains, are offered to people in their diet. However, it is an issue of concern in selecting and/or recommending fermented products like kefir for consumption, whether industrial kefir products have probiotic values similar to those of traditional kefir. Therefore, this study quantitatively determined the microbial composition of industrial kefir products offered to the market and traditional kefir samples, both at the dates of purchase and expiration date, and to reveal the microbial diversity of these products. This study also compared the fruit and plain kefir products in terms of their probiotic content and $\mathrm{pH}$ values.

Kefir contains lactic acid bacteria (Lactobacilli, Lactococci, Leuconostocs, and Streptococci), yeast (Candida spp., Kluyveromyces spp., Saccharomyces spp., Torulopsis spp., Zygosaccharomyces spp.), and rarely acetic acid bacteria (Acetobacter spp.) (De Moreno de LeBlanc et al., 2006; Farnworth, 2005; Güzel-Seydim et al., 2011; Motaghi et al., 1997; Witthuhn et al., 2004). Several studies report the benefits of this product with the rich microbial composition (Cevikbas et al., 1994; Vinderola et al., 2006; Maeda et al., 2004; Urdaneta et al., 2007). In particular, lactic acid bacteria's therapeutic effect like reducing cholesterol level, improving immunity, and reducing gastrointestinal symptoms has been emphasized for many years (Tamai et al., 1996; Perdigon et al., 2001; Alm, 1982; Rosa et al., 2017). In the kefir samples examined in this study, Lactobacillus paracasei, Lactobacillus casei, Lactococcus lactis, Leuconostoc mesenteroides, Leuconostoc spp, Streptococcus sp., and Candida kefyr were isolated from kefir samples included in the study. Acetic acid bacteria were not isolated in any kefir samples. We can conclude from results that the kefir products examined in this study were found to have enough probiotic variety to constitute the kefir flora.

Several studies have highlighted the microorganism diversity of kefir produced using traditional methods (Oner et al., 2010, Guzel-Seydim et al., 2005; Walsh et al., 2016). However, this study determined relatively fewer microorganism varieties in the kefir samples produced using the traditional method. Note that factors such as milk, type of grain, incubation time, or environmental conditions may have affected kefir products' microbial diversity. Nevertheless, industrial kefirs with an average shelf life of 20 days had a microbial variety that could provide the expected benefits of kefir in both measurement times.

One study determined that the number of microorganisms remained constant in the first 15 days of kefir's storage time made from cow milk (Oner et al., 2010). Similarly, Leite et al. (2013) observed no change in the number of lactic acid bacteria and yeasts. However, Irigoyen et al. (2005) observed that the number of Lactobacillus and Lactococcus decreased in the 2nd week in both series prepared by adding $1 \%$ and $5 \%$ kefir grains, respectively. In this study, the number of Lactococcus decreased in the second measurement of plain kefir samples $(\mathrm{p}<0.05)$. Another similar study found a lower number of Lactococcus in the second week of the study (Irigoyen et al. 2005). They found that the counts of yeast and acetic acid bacteria remained constant during cold storage, and the count of LAB decreased in the 7-14 days of storage (Irigoyen et al. 2005). Montanuci et al. (2012) reported that the number of yeasts, acetic acid bacteria, and Leuconostoc increased or remained unchanged at the end of the storage period, but the total LAB and Lactococcus population decreased by 1 to 2 logs or remained unchanged during the storage. In another study, the LAB number of five commercial Norwegian kefir samples decreased in the first 4 weeks of storage, while the number of yeasts increased during the storage period (Grønnevik et al., 2011). This study found a statistically significant difference between the counts of Lactobacillus, Lactococcus, Leuconostoc, and Streptococcus at two different times $(\mathrm{p}<0.05)$, where the average value in all four parameters was lower at the end of the expiration date than at the beginning of the production date (Table 2). Despite this numerical decrease, all industrial kefir samples complied with the definition of kefir according to Food Codex in both measurements, which suggests that there is no risk/harm in consuming these products until expiry date.

In this study, the yeast was found only in 2 of the 10 samples, including 1 plain and 1 fruit kefir products of the same trademarks. The number of yeasts increased in only a sample with yeast (Table 2). Loretan et al. (2003) determined the number of yeasts in a home-made kefir sample as $8 \log 10 \mathrm{cfu} / \mathrm{mL}$ but found no yeast in it. Contrary to previous studies, the fact that the yeast was not isolated from the kefir samples produced using the traditional method in this study suggests that the stability of microbial content in kefirs produced using the traditional method could not be achieved. Note that different microbial contents can be obtained by changing the conditions such as grain, milk, time, and temperature.

One study, conducted in Ireland, has examined the microbial composition of six kefir samples and found a similarity in terms of Lactobacillus, Lactococcus, Leuconostoc, acetic acid bacteria, and yeast numbers (Rea et al. 1996). Similarly, this study compared fruit and plain kefir samples in terms of the number of lactic acid bacteria at two different times and found no statistically significant difference between them. The products sold in the market were compared with their counterparts at two different times and observed to have similar LAB numbers. In this context, there is no risk for individual preferences to come to the fore in selecting fruit or plain kefir samples. However, sugar additives should be considered in selecting kefir in bodyweight management or in the presence of diseases such as diabetes mellitus, where even simple sugar intake should be taken into consideration.

Leite et al. (2013) found that the $\mathrm{pH}$ value of Brazilian kefir decreased in the period from fermentation to the expiration date. Another study has emphasized that the $\mathrm{pH}$ value continuously decreases in the cold storage period after fermentation (Guzel-Seydim et al., 2005). Similarly, this study determined that the $\mathrm{pH}$ values of industrially produced kefir samples decreased in the storage process but found a statistically significant decrease only in plain kefir samples $(\mathrm{p}<0.05)$. The $\mathrm{pH}$ values of plain and fruit 
kefir samples were compared simultaneously and found to be stable, suggesting no statistically significant difference.

This study has some limitations. Only one kefir sample was prepared by the traditional method with one type of kefir grain, and industrial kefir samples included pasteurized milk of only two brands. Therefore, it will be useful for further studies to examine more than one sample by preparing kefirs with the traditional method using pasteurized milk of different brands and grains. Besides, no standardization has been achieved regarding the supply of industrial products in the market, and there was a possibility that the products were not offered for sale in similar conditions (cold chain compatibility, date of arrival, storage conditions, etc.) before purchase, both of which limited this study. However, as a consumer does not have a chance to follow this chain, the existing conditions can be considered suitable.

Microbiological and chemical changes in the process from kefir production to consumption contribute to its unique taste, creating its own unique flavor. The microbiological properties of kefir vary depending on grain to milk ratio, incubation, or storage conditions. This study found that the number of microorganisms in industrial kefir samples decreased at expiration dates. There was a significant decrease in the number of Lactococcus only in plain kefir samples sold in the market. No differences were observed between fruit and plain kefir samples in terms of the number of lactic acid bacteria measured at two different times, and all kefir samples examined had probiotic properties. Therefore, there is no difference in preferring plain or fruit kefir to benefit from these bacteria's positive health effects (on gastrointestinal disorders, metabolic diseases, etc.). However, notably the number of microorganisms was relatively lower at the expiration date. Depending on these results, awareness studies on the dates when kefirs are offered to the market should be conducted. Besides, nutritionists should provide detailed information about label reading when they include kefir in nutrition plans. They should also emphasize that individual differences in the selection of flavored or plain kefir do not affect the benefits expected from kefir. Furthermore, there is a need to increase the number of scientific studies on microbiological changes in fermented products and to share the importance of this issue with consumers.

\section{References}

Ahmed Z, Wang Y, Ahmad A, Khan, ST, Nisa M, Ahmad H and Afreen A. 2013. Kefir and health: a contemporary perspective. Crit Rev Food Sci Nutr., 53: 422-434. doi: 10.1080/10408398.2010.540360.

Alm L. 1982. Effect of fermentation on lactose, glucose, and galactose content in milk and suitability of fermented milk products for lactose intolerant individuals. J Dairy Sci., 65: 46352. doi: 10.3168/jds.S0022-0302(82)82198-X.

Cevikbas A, Yemni E, Ezzedenn FW, Yardimici T, Cevikbas U and Stohs SJ. 1994. Antitumoural antibacterial and antifungal activities of kefir and kefir grain. Phytother Res., 8(2): 78-82. doi: 10.1002/ptr.2650080205.

De Moreno de LeBlanc A, Matar C, Farnworth ER and Perdigon G. 2006. Study of cytokines involved in the prevention of a murine experimental breast cancer by kefir. Cytokine, 34: 1-8. doi:10.1016/j.cyto.2006.03.008.
Farnworth ER. 2005. Kefir a complex probiotic. Food Sci Technol Bull.: Functional Foods, 2: 1-17. doi: 10.1616/1476-2137.13938.

Grønnevik H, Falstad M and Narvhus JA. 2011. Microbiological and chemical properties of Norwegian kefir during storage. Int Dairy J., 21: 601-606. doi: 10.1016/j.idairyj.2011.01.001.

Guzel-Seydim Z, Wyffels JT, Seydim AC and Greene AK. 2005. Turkish kefir and kefir grains: microbial enumeration and electron microscobic observation. Int. J. Dairy Technol., 58(1): 25-29. doi:10.1111/j.1471-0307.2005.00177.x

Guzel-Seydim ZB, Kok-Tas T, Greene AK and Seydim AC. 2011. Review: Functional properties of kefir. Crit Rev Food Sci Nutr., 51: 261-268. doi:10.1080/10408390903579029.

Hassan FH, Golnar R, Mohammad RF, Mitra M and Mitra S. 2012. Evaluation of wound healing activities of kefir products. Burns, 38(5): 719-723. doi: 10.1016/j.burns.2011.12.005.

Heyman M. 2000. Effect of lactic acid bacteria on diarrheal diseases. $J$ Am Coll Nutr., 19: 137-146. doi: 10.1080/07315724.2000.10718084.

Irigoyen A, Arana I, Castiella M, Torre P and Ibanez FC. 2005. Microbiological, physicochemical, and sensory characteristics of kefir during storage. Food Chem., 90(4): 613-620. doi: 10.1016/j.foodchem.2004.04.021.

John SM and Deeseenthum S. 2015. Properties and benefits of kefir-A review. Songklanakarin J Sci Technol., 37(3), 275-282.

Leite AMO, Leite DCA, Del Aguila EM, Alvares TS, Peixoto RS, Miguel MAL, Silva JT and Paschoalin VMF. 2013. Microbiological and chemical characteristics of Brazilian kefir during fermentation and storage processes. J Dairy Sci., 96(7): 4149-4159.

Loretan T, Mostert JF and Viljeon BC. 2003. Microbial flora associated with South African house hold kefir. S Afr J Sci., 99: 92-94.

Maeda H, Mizumoto M, Suzuki K and Tsuji K. 2005. Effects of Kefiran-Feeding on Fecal Cholesterol Excretion, Hepatic Injury and Intestinal Histamine Concentration in Rats. Biosci and Microflora., 24(2): 35-40. doi: 10.12938/bifidus.24.35.

Maeda H, Zhu X, Omura K, Suzuki S and Kitamura S. 2004. Effects of an exopolysaccharide (kefiran) on lipids, blood pressure, blood glucose, and constipation. Biofactors, 22: 197200. doi: 10.1002/biof.5520220141.

Montanuci FD, Pimentel TC, Garcia S and Prudencio SH. 2012. Effect of starter culture and inulin addition on microbial viability, texture, and chemical characteristics of whole or skim milk kefir. Cienc tecnol aliment., 32: 850-861. doi: 10.1590/S010120612012005000119.

Motaghi M, Mazaheri M, Moazami N, Farkhondeh A, Fooladi MH and Goltapeh E.M. 1997. Short communication: Kefir production in Iran. World J Microbiol Biotechnol., 13: 579-581. doi:10.1023/A:1018577728412.

Oner Z, Karahan AG and Cakmakci ML. 2010. Effects of different milk types and starter cultures on kefir. Gida 35: 177182.

Otles S and Cagindi O. 2003. Kefir: A probiotic dairycomposition, nutritional and therapeutic aspects. Pakistan J Nutr., 2(2): 54-59. doi: 10.1590/S1517-83822013000200001.

Perdigon G, Nader de Macias E, Alvarez S, Medici M, Oliver G and de Ruiz Holgado AP. 1986. Effect of mixture on Lactobacillus casei and Lactobacillus acidophilus administered orally on the immune system in mice. J Food Prot., 49: 986-989. doi: 10.4315/0362-028X-49.12.986. 
Prado MR, Blandón LM, Vandenberghe LP, Rodrigues C, Castro GR, Thomaz-Soccol V and Soccol C.R. 2015. Milk kefir: composition, microbial cultures, biological activities, and related products. Front Microbiol.,6: $1177 . \quad$ doi: 10.3389/fmicb.2015.01177.

Rea MC, Lennartsson T, Dillon P, Drinan FD, Reville, Heapes M and Cogan TM. 1996. Irish kefir-like grains: their structure, microbial composition and fermentation kinetics. $J \quad A p p l$ Microbiol., 81(1): 83-94.

Reid G, Jass J, Sebulsky MT and McCormick JK. 2003. Potential uses of probiotics in clinical practice. Clin Microbiol Rev., 16: 658-72. doi: 10.1128/CMR.16.4.658-672.2003.

Rosa DD, Dias MM, Grześkowiak ŁM, Reis SA, Conceição LL and Maria do Carmo GP. 2017. Milk kefir: nutritional, microbiological and health benefits. Nutr Res Rev., 30(1), 82-96.

Tamai Y, Yoshimitsu N, Watanabe Y, Kuwabara Y and Nagai S. 1996. Effects of milk fermented by culturing with various lactic acid bacteria and a yeast on serum cholesterol level in rats. $J$ Ferment Bioeng., 81(2): 181-182.

Urdaneta E, Barrenetxe J, Aranguren P, Irigoyen A, Marzo F and Ibanez FC. 2007. Intestinal beneficial effects of kefirsupplemented diet in rats. Nutr Res., 27(10): 653-658. doi: 10.1016/j.nutres.2007.08.002.
Vinderola CG, Duarte J, Thangavel D, Perdigón G, Farnworth E and Matar C. 2005. Immunomodulating capacity of kefir. J Dairy Res., 72(2): 195-202. doi: 10.1017/S0022029905000828.

Vinderola CG, Perdigon G, Duarte J, Farnworth E and Matar C. 2006. Effects of kefir fractions on innate immunity. Immunobiology, 211:149-156. doi: 10.1016/j.imbio.2005.08.005.

Walsh AM, Crispie F, Kilcawley K, O’Sullivan O, O’Sullivan MG, Claesson MJ and Cotter PD. 2016. Microbial succession and flavor production in the fermented dairy beverage kefir. Msystems 1(5): e00052-16.

Witthuhn RC, Schoeman T and Britz TJ. 2004. Isolation and characterization of the microbial population of different South African kefir grains. Int. J. Dairy Technol., 57: 33-37. doi:10.1111/j.1471-0307.2004.00126.x.

Yüksekdağ ZN, Beyatli Y and Aslim B. 2004. Determination of some characteristic coccoid forms of lactic acid bacteria isolated from Turkish kefirs with natural probiotic. LWT-Food Sci Technol., 37(6): 663-667. doi: 10.1016/j.lwt.2004.02.004. 